\title{
Oral Modeling of an Adenovirus-Based Quadrivalent Influenza Vaccine in Ferrets and Mice
}

\author{
Ciaran D. Scallan · Jonathan D. Lindbloom · Sean N. Tucker
}

Received: February 10, 2016 / Published online: April 12, 2016

(C) The Author(s) 2016. This article is published with open access at Springerlink.com

\begin{abstract}
Introduction: Oral vaccines delivered as tablets offer a number of advantages over traditional parenteral-based vaccines including the ease of delivery, lack of needles, no need for trained medical personnel, and the ability to formulate into temperature-stable tablets. We have been evaluating an oral vaccine platform based on recombinant adenoviral vectors for the purpose of creating a prophylactic vaccine to prevent influenza, and have demonstrated vaccine efficacy in animal models and substantial immunogenicity in humans. These studies have evaluated monovalent vaccines to date. To protect against the major circulating $\mathrm{A}$ and $\mathrm{B}$ influenza strains, a multivalent influenza vaccine will be required.
\end{abstract}

Enhanced content To view enhanced content for this article go to http://www.medengine.com/Redeem/ 5E84F06034FA1B2F.

C. D. Scallan · J. D. Lindbloom · S. N. Tucker $(\square)$ Vaxart, Inc., South San Francisco, CA, USA e-mail: stucker@vaxart.com
Methods: In this study, the immunogenicity of orally delivered monovalent, bivalent, trivalent, and quadrivalent vaccines was tested in ferrets and mice. The various vaccine combinations were tested by blending monovalent recombinant adenovirus vaccines, each expressing hemagglutinin from a single strain. Human tablet delivery was modeled in animals by oral gavage in mice and by endoscopic delivery in ferrets.

Results: We demonstrated minimal interference between the various vaccine vectors when used in combination and that the oral quadrivalent vaccine compared favorably to an approved trivalent inactivated vaccine.

Conclusion: The quadrivalent vaccine presented here produced immune responses that we predict should be capable of providing protection against multiple influenza strains, and the platform should have applications to other multivalent vaccines.

Funding: Vaxart, Inc.

Keywords: Ferret; Influenza; Mouse; Oral; Vaccination 


\section{INTRODUCTION}

Influenza virus infections are a major cause of acute seasonal respiratory illness, which causes significant morbidity and mortality. Vaccination is an effective way of reducing the numbers of infected individuals provided there is a good match between the vaccine and the circulating strains. Every February in the Northern Hemisphere the circulating strains for the next season are chosen by the World Health Organization, which allows approximately 6 months for sufficient vaccine to be generated. Annual influenza vaccines need to provide protection against the predominant influenza strains that are present in each season's outbreaks. Since 1977, A/H1N1, A/H3N2, and B viruses are the major virus types that are in circulation. Choosing only one B strain was not always accurate as indicated during the period of 2001-2010 in the USA when the trivalent influenza strain only partially matched the circulating B strain 50\% of the time [1]. Licensed vaccines today use three (two $A$ and one B) or four (two A and two B) strains that are predicted to be circulating in the population. The additional $B$ strain in the quadrivalent vaccine is due to the fact that there are two B lineages (B/ Yamagata and B/Victoria) currently in co-circulation and there is limited cross protection between strains from each lineage [2]. In a study using UK data, it was estimated that the inclusion of an additional B strain to generate a quadrivalent vaccine could be expected to reduce the number of influenza cases by 17,088 and deaths by 168 in the first year of use [3]. Although adding four strains may reduce the possibility of $\mathrm{B}$ strain mismatch, strain mismatch can still occur as was the case for the 2014/15 Northern Hemisphere influenza season, where the predominant H3N2 strain drifted [34], with the result that the vaccine was only partially protective (vaccine effectiveness of only 23\%) [35]. While four strains may be better than one, strain selection is by no means perfect.

An additional issue that arises with multivalent vaccines is the possibility of immunological interference effects between the individual vaccine components. Interference can result in a diminished or an enhanced response to one component in the presence of another. This has been documented for live attenuated influenza vaccines [4], and where it occurs, dosing may have to be modified to counteract this interference [5]. As new quadrivalent vaccines are developed, interference effects need to be studied for each new vaccine.

We have been developing an oral-based adenovirus platform for vaccine delivery. The vaccine platform consists of a replication-incompetent adenovirus vector bearing two expression cassettes that express a vaccine antigen and a double-stranded RNA (dsRNA) adjuvant [6]. The vaccine antigen that we have chosen for the influenza vaccine is influenza hemagglutinin (HA), an envelope glycoprotein involved in receptor binding and viral fusion. Antibodies that block the ability of HA to bind to target cells, as measured by the HA inhibition (HAI) titer, have been found to correlate with protection [7]. This oral vaccine approach has a number of advantages over traditional influenza vaccines. These include ease of manufacturing, using standard recombinant techniques and established viral purification methods; ease of delivery without needles or other devices that need qualified medical support; long-term stability at room temperature negating the need for cold storage. This oral platform also generates minimal anti-vector immunity thus permitting revaccination and reuse [6]. We have generated HA-based recombinant adenovirus (rAd) vaccine vectors to individual influenza strains and 
demonstrated either protection in small animals [6] or immunogenicity in humans [8, 9]. Results from these studies demonstrate the ability of the approach to generate neutralizing antibody responses to influenza, with additional advantages such as antibody durability and $\mathrm{T}$ cell responses shown in the studies as well. Given the commercial need for a seasonal vaccine that covers more than one strain, we have explored the utility of our platform to vaccinate against multiple influenza strains simultaneously and have evaluated whether significant interference exists between the vaccine antigens. Here, we model oral tablet delivery by measuring the ability of ferrets to mount immune responses to multiple strains following intestinal delivery to a single site and establish a potential strategy for eventual vaccine commercialization.

\section{METHODS}

\section{Cell Lines, Trivalent Vaccine}

HEK293 cells were propagated in Dulbecco's Modified Eagles Medium (DMEM) plus GlutaMax $^{\mathrm{TM}}$ (Invitrogen, Carlsbad, CA, USA) supplemented with 5\% bovine serum (BS) and antibiotics (10 units/mL penicillin and $10 \mu \mathrm{g} / \mathrm{mL}$ streptomycin). Trivalent inactivated influenza vaccine (TIV), Fluzone ${ }^{\circledR}$ (Sanofi Pasteur, Inc.), was composed of the strains: A/California/07/09; A/Victoria/201/09 (A/Perth/16/09-like virus); $\mathrm{B} /$ Brisbane/60/08, and used as a comparative vaccine in the ferret quadrivalent study evaluation.

\section{Adenovirus Propagation and Characterization}

E1, E3 deleted rAd subtype-5 vectors were produced using the AdEasy ${ }^{\mathrm{TM}}$ (Qbiogene, Quebec, Canada) kit. The original non-codon optimized sequences for the different HA genes used in this study were obtained from GenBank (NCBI, Bethesda, MD, USA). The following Genbank accession numbers were used for the HA constructs; FJ966082 for A/California/04/2009 (H1N1), GQ293081 for A/Perth/16/09 (H3N2), CY11515.1 for B/Brisbane/60/2008 (B/Victoria) and KC306166.1 for B/Wisconsin/01/2010. All HA genes were human codon optimized and synthesized by Blue Heron Biotechnology (Bothel, WA, USA). The HA gene was synthesized with flanking Kpn I sites, which facilitated cloning into a pShuttle-cytomegalovirus (CMV) vector (Qbiogene) that also contained a human ß-globin intron and the bovine growth hormone polyadenylation(A) signal. This construct also expressed a molecular adjuvant as a short dsRNA hairpin under control of a second CMV promoter and which utilized a minimal synthetic polyadenylation(A) signal. The adjuvant sequence was made by annealing overlapping oligonucleotide primers and has been described previously [6]. The shuttle vectors were propagated in NeB10 $\beta$ cells (New England Biolabs, Beverly, MA, USA), and screened by restriction digests and HA-specific polymerase chain reaction primers. Selected pShuttle clones were used to generate adenovirus stocks by recombination in BJ5183-AD1 bacteria (Stratagene, La Jolla, CA, USA) and transfection into HEK293 cells as previously described [6]. The $\mathrm{HA}$ vaccine vectors were purified by $\mathrm{CsCl}$ centrifugation and dialyzed into $20 \mathrm{mM}$ Tris $\mathrm{pH}$ 8.0, $25 \mathrm{mM} \mathrm{NaCl}, 2.5 \%$ glycerol. The viral titer (IU/mL) was determined by immunostaining using a rabbit anti-adenovirus polyclonal (Abcam, Cambridge, MA, USA) as the primary antibody, an anti-rabbit HRP secondary (Bethyl Laboratories, Montgomery, TX, USA) antibody and the color substrate 3,3'-diaminobenzidine (DAB) for signal detection. 


\section{Animal Experiments}

Animal research was approved by the Institutional Animal Care and Use Committees (IACUC) at Vaxart (South San Francisco, CA, USA) and Southern Research Institute (SRI) (Birmingham, AL, USA). Six- to seven-week-old BALB/c mice were acquired from Simonsen Laboratories (Gilroy, CA, USA) and vaccinated perorally (p.o.) similar to that described by other investigators [10, 11]. Briefly, $0.2 \mathrm{~mL}$ of $7.5 \%$ sodium bicarbonate was given by $24 \mathrm{G}$ feeding tube (Fine Science Tools, Foster City, CA, USA) followed less than a minute later with rAd in $0.2 \mathrm{~mL}$ of phosphate-buffered saline (PBS). Adenovirus is $\mathrm{pH}$ sensitive, so stomach neutralization protects against acid degradation. Intra-nasal (i.n.) vaccinations were performed similar to that described by Moore et al. [12]. For oral dosage, $1.0 \times 10^{7} \mathrm{IU}$ per mouse was administered at day 0 and week 4 , and for i.n. dosing, $2 \times 10^{6} \mathrm{IU} /$ mouse was administered at day 0 , unless specifically stated. Plasma samples were acquired by cheek-pouch lancet (Medipoint, Mineola, NY, USA) at several time-points post-vaccination. For i.n. vaccination and for plasma harvesting, mice were anesthetized by isoflurane inhalation using a certified vaporizer (VetEquip, Livermore, CA, USA). Mouse experiments were performed once, but the results are representative of several similar experiments where vectors have been blended.

Fitch ferrets (Triple F Farms, Sayre, PA, USA) were used in the ferret studies. Ferrets were prescreened to select those animals negative for HAI titers to the given strains. For the bivalent B vaccine evaluation, male ferrets (1052-1856 g) of 20-24 weeks of age were used with $N=8$ for oral groups, $N=3$ for intra-muscular (i.m.; with the exception of $N=2$ for the recombinant adenoviral vector expressing $\mathrm{HA}$ from
B/Brisbane/60/08 where an animal died prematurely at day 0 due to non-vaccine issues). For the quadrivalent study, 16-17-week-old male and female ferrets (677-1341 g) were used. Each group (Groups $1-7, n=10$; Group 8, $n=4$ ) used equal numbers of males and females. Each ferret experiment was performed once with $N=8-10$ to reduce total animal use. Prior to p.o. vaccination, ferrets were fasted between 1 and $4 \mathrm{~h}$ to allow ample time for the gastric emptying. Viral vectors were delivered to the small intestine via a bronchoscope (used as an endoscope) as previously described [6]. $1 \times 10^{9} \mathrm{IU}$ of rAd vectors were administered in $1 \mathrm{~mL}$ of $\mathrm{PBS}$ on weeks 0 and 4. This dose represents approximately $1: 10$ or $1: 100$ of a human effective dose as described by Liebowitz et al. [8] and Peters et al. [9]. For blended vectors, the vaccines were first mixed and then $1 \times 10^{9} \mathrm{IU}$ of each vector component was administered. For i.m. vaccinations, animals were dosed with $0.5 \mathrm{~mL}$ of either an rAd vector vaccine or TIV (Fluzone; at one full human dose) injected into the hind limb muscle.

\section{HA Assays}

Total anti-HA antibodies were measured similarly as previously described [6]. Briefly, microtiter plates (Nunc MaxiSorp ${ }^{\circledR}$, eBioscience, Inc., San Diego, CA, USA) were coated in $1 \times$ carbonate buffer $(0.1 \mathrm{M}$ at $\mathrm{pH} 9.6)$ with $1.0 \mu \mathrm{g} /$ $\mathrm{mL}$ of a baculovirus-expressed influenza $\mathrm{HA}$ protein (Protein Sciences Corporation, Meriden, CT, USA). Plates were incubated overnight at $4{ }^{\circ} \mathrm{C}$ in a humidified chamber, then blocked in PBS $+0.05 \%$ Tween 20 (PBST) $+1 \%$ BS albumin (BSA) solution for $1 \mathrm{~h}$ before washing. Plasma samples were serially diluted in PBST. After a 2-h incubation, plates were washed $5 \times$ with PBST. Antibodies were then added, either as a mixture 
of anti-mouse immunoglobulin G1 (IgG1)-horseradish peroxidase (HRP) and anti-mouse IgG2a-HRP (Bethyl Laboratories), or alternatively, anti-mouse IgA-HRP (Bethyl Laboratories) for mucosal samples. Each secondary antibody was used at a 1:5000 dilution and incubated for $1 \mathrm{~h}$. Plates were washed at least five times. Antigen-specific antibodies were detected with 3,3',5,5'-tetramethylbenzidine (TMB) substrate (Rockland, Gilbertsville, PA, USA) using a microplate reader. $\mathrm{H}_{2} \mathrm{SO}_{4}$ was used as a stop solution, and the plates were read at $450 \mathrm{~nm}$ on an $\mathrm{EMax}^{\circledR}$ enzyme-linked immunosorbent assay (ELISA) plate reader (Molecular Devices, Sunnyvale, CA, USA). Average mouse antibody titers were reported as the reciprocal dilution giving an absorbance value greater than the average background plus two standard deviations (SDs) unless otherwise stated. Ferret antibody responses to HA were measured by a similar ELISA protocol with the exception that the secondary antibody was anti-Ferret IgG-HRP (Bethyl Laboratories). Ferret sera antibody titers were calculated by performing the ELISA on both the day 0 and the test samples (days 28 and 56) on the same plate and a titer was assigned as the reciprocal of the highest dilution of the test sample where the value was greater than the corresponding day 0 dilution by 0.04 or greater. The absorbance value also had to be greater than the average background (based on blocking buffer alone) plus two SDs.

HAI titers were measured by Focus Diagnostics (Cypress, CA, USA). The HAI protocol was followed as described before [13] using four HA units of the relevant viruses. In brief, test sera were first treated with receptor-destroying enzyme (RDE; Accurate Chemical \& Scientific, Westbury, NY, USA) at a $1: 3$ ratio at $37^{\circ} \mathrm{C}$ for $18-20 \mathrm{~h}$. The RDE was then inactivated by incubation at $56^{\circ} \mathrm{C}$ for $30 \mathrm{~min}$. Six volumes of saline was then added to generate a final sera dilution of 1:10. Influenza viruses were diluted to a working stock of $4 \mathrm{HA}$ units $/ 25 \mu \mathrm{L}$. Washed turkey red blood cells (Lampire Biological Products, Pipersville, PA, USA) were prepared by washing $4 \times$ in PBS and were resuspended in PBS at a final volume of $0.45 \%$. A $1: 2$ dilution series of the $1: 10$ pre-diluted sera was prepared in columns 1-10 of a V-shaped polystyrene microtiter plate with row 1 undiluted, rows 2-8 diluted in PBS, $25 \mu \mathrm{L}$ PBS well. Columns 11 and 12 contained negative sera and no sera (PBS only) respectively. $25 \mu \mathrm{L}$ of an influenza stock (4 HA units $/ 25 \mu \mathrm{L}$ ) was added per well and incubated for $1 \mathrm{~h}$ at room temperature. Fifty microliters of the $0.45 \%$ RBC was added and incubated for 45 min. Agglutination was compared between the test and PBS wells. HAI titers were assigned as the reciprocal of the highest sera dilution that completely prevented RBC agglutination. The influenza $\mathrm{B}$ viruses were egg derived and pretreated with ether (Virapur, San Diego, CA, USA) prior to use in the HAI assay [14]. Both the A/Perth/16/09 and A/California/07/09 strains used were Madin-Darby canine kidney (MDCK) derived.

Microneutralization (MN) assays for both the blended B and quadrivalent ferret studies were performed by SRI following a protocol described by Rowe et al. [15]. In brief, sera were inactivated at $56^{\circ} \mathrm{C}$ for $30 \mathrm{~min}$ and twofold serial dilutions were made in 1\% BSA/DMEM/ $1 \mathrm{X}$ penicillin/streptomycin. The diluted sera $(50 \mu \mathrm{L})$ was mixed with an equal volume of influenza viruses at $2 \times 10^{3}$ tissue culture infectious dose 50 (TCID50)/mL and incubated for $18 \mathrm{~h}$ at $37^{\circ} \mathrm{C}$. Control wells of virus plus dilution buffer or dilution buffer alone were included on each plate. $100 \mu \mathrm{L}$ of MDCK cells at $1.5 \times 10^{5} / \mathrm{mL}$ was added to each well. The plates 
were incubated for $18 \mathrm{~h}$ at $37^{\circ} \mathrm{C}$ and $5 \% \mathrm{CO}_{2}$. The monolayers were washed with PBS and fixed in cold $80 \%$ acetone for $10 \mathrm{~min}$. The presence of viral protein was detected by an anti-influenza A nucleoprotein (NP) ELISA using an anti-NP antibody (Mab8251, Millipore, Billerica, MA, USA). The detecting antibody was an HRP-goat anti-mouse antibody (Kirkegaard \& Perry Laboratories, Gaithersburg, MD, USA) at a 1:2000 dilution and the substrate used for detection was o-phenylenediamine dihydrochloride $\quad(0.5 \mathrm{mg} / \mathrm{mL}$ in $0.05 \mathrm{M}$ phosphate citrate buffer, $\mathrm{pH}$ 5.0/0.03\% sodium perborate). A titer was assigned as the reciprocal of the highest dilution that neutralized greater than $50 \%$ of the virus-only control. Values less than 20 were assigned a value of 10 . SRI used egg-derived viruses obtained from the Centers for Disease Control and Prevention. No MN assay was available for the H3N2 strain as SRI had previously established that ferret sera generated too high a background with this H3N2 strain.

\section{Statistical Analysis}

Significance between groups was determined by Mann-Whitney analysis using Prism software or by Fisher's exact test (http://vassarstats.net/ tab2x2.html). $P$ values $<0.05$ were considered significant.

\section{RESULTS}

Monovalent and Blended Vectors Induce HA-Specific Immunity in BALB/c Mice Vaccinated via the Oral and Intra-Nasal Routes

Monovalent adenovirus vector vaccines were generated for HAs representing the major viral strains circulating in the 2010/11 Northern Hemisphere. Figure 1 shows a schematic representation of the transgene and adjuvant cassette. Expression of each of the HAs was confirmed by Western blotting of infected cell lysates with strain-specific antibodies (data not shown).

To first examine the potential for blending, adenoviruses were initially evaluated in BALB/c mice, using blended monovalent vectors compared to unblended monovalent vectors, and using multiple routes of administration. A trivalent configuration was tested first, with the anti-HA IgG response measured by ELISA (Fig. 2a). Oral gavage was used to deliver the vaccine to mice p.o. The blend tested in this experiment consists of a blend of $1 \times 10^{7} \mathrm{IU}$ of vectors expressing H1/California/04/09, H3/ Perth/16/09, and B/Brisbane/60/08 HA antigens. These were compared to the vectors tested alone and a naïve group of animals and demonstrated that the blended vectors behaved comparably to the individual vectors. The response to B/Brisbane/60/08 in the blend did trend lower than for the animals treated with the monovalent vaccine, but the difference was not statistically significant $(P=0.361)$. There was very little cross strain reactivity when sera from the monovalent vectors was tested against HA antigens from the other strains indicating that antibodies to the specific HA in the trivalent blended sera were generated by each individual HA component.

The i.n. route was evaluated as an additional mucosal route of delivery, and has the advantage of less intra-group variability compared to gavage delivery in mice. This allowed the evaluation of additional permutations on the blend data from p.o. experiment described before. The two blending permutations from the standard 1:1:1 blend that were tested were a 1:10:1 blend where the 
Transgene

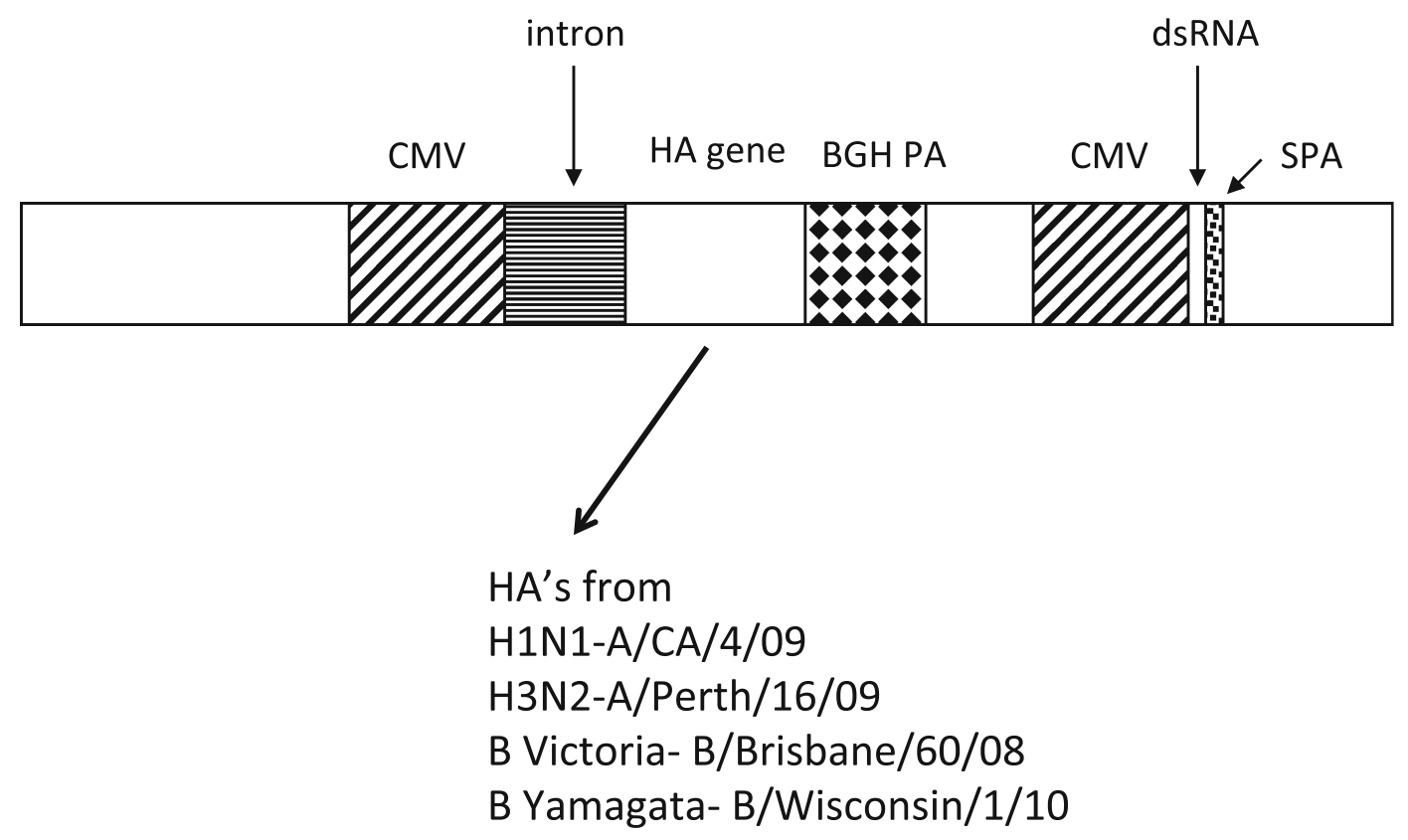

\section{Adjuvant}

Fig. 1 Schematic of vaccine expression cassettes. Codon optimized HA genes from individual seasonal influenza strains were cloned into an expression cassette driven by a CMV promoter and upstream of the region coding for a dsRNA sequence also under the control of a CMV promoter.

B/Brisbane/60/08 HA vaccine was dosed ten times higher than the $\mathrm{H} 1$ and $\mathrm{H} 3$ vaccines, and a group in which the three vaccine components (1:1:1) were dosed separately with $24 \mathrm{~h}$ between doses (Fig. 2b). Again, there was no significant difference between the blended and individual vaccine groups in terms of immune responses to each of the HAs $(P=0.2-0.99$ by Mann-Whitney analysis). If anything, the trivalent trended higher for IgG antibody responses to influenza $B$ than the monovalent $(P=0.2)$. The trivalent group with ten times higher B vaccine dose did have a higher but not significantly different immune response to the (1:1:1) trivalent group, or to the monovalent B group $(P=0.11$ and 0.11 by Mann-Whitney analysis, respectively). Vaccine staggering did not appear to affect the immune response
$C M V$ Cytomegalovirus, $B G H P A$ Bovine growth hormone polyadenylation(A) signal, $d s R N A$ Double-stranded RNA adjuvant, $H A$ Hemagglutinin, intron Beta globin intron, $S P A$ Synthetic polyadenylation(A) signal

compared to the blended group dosed with all constituents at the same time.

Before beginning a quadrivalent study, an additional experiment looking at cross reactivity of $\mathrm{B} /$ Victoria and $\mathrm{B} /$ Yamagata $\mathrm{HA}$ vaccines was performed in mice. Animals were dosed with either Ad-B/Brisbane/60/08 (Ad-B/ Bris) (Victoria), Ad-B/Wisconsin/1/10 (Ad-B/ Wis) (Yamagata) or a blend of the two. All vectors were dosed with each vector at $2 \times 10^{6} \mathrm{IU} /$ animal. The Ad-B/Bris group induced a geometric mean titer (GMT) of $5.82 \times 10^{3} \pm 4.2 \times 10^{4}$ to $\mathrm{B} /$ Brisbane $/ 60 / 08$ and $1.12 \times 10^{3} \pm 1.93 \times 10^{3}$ to $\mathrm{B} /$ Wisconsin/1/10 indicating substantial cross reactivity (Fig. 3). As was the case for Ad-B/Bris, Ad-B/Wis induced a GMT of $6.31 \times 10^{4} \pm 3.97 \times 10^{4} \quad$ to B/Wisconsin/1/10 and $1.63 \times 10^{3} \pm 4.46 \times 10^{3}$ 


\section{A p.o. delivery}

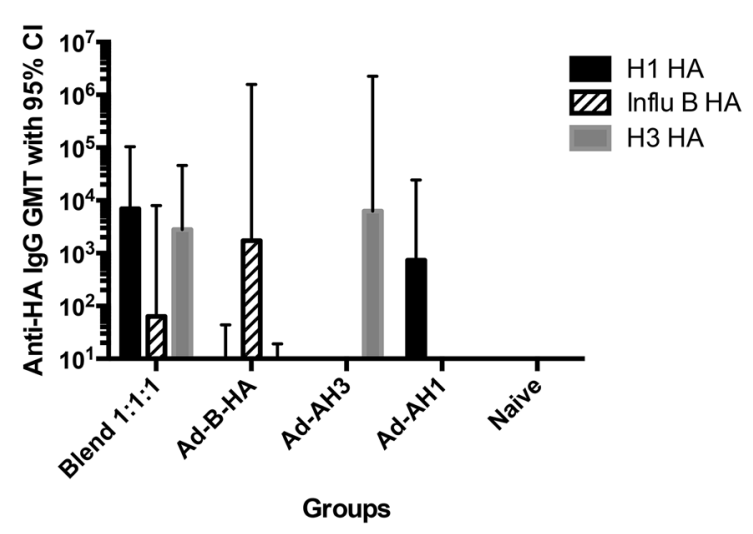

B i.n. delivery

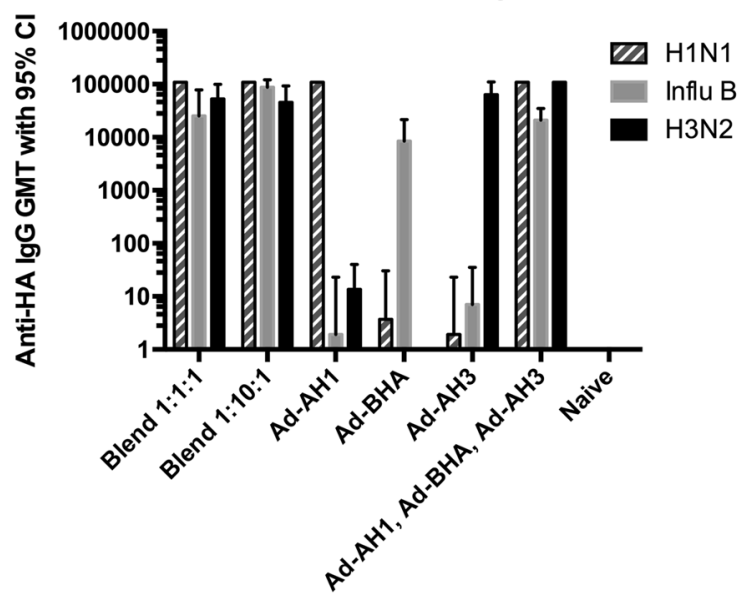

Fig. 2 a Oral administration of Ad-HA vaccines in BALB/ c mice. $1 \times 10^{7} \mathrm{IU}$ of each vector was either administered as a blend, or individually. Vaccines were administered at time 0 and week 4. Total anti-HA IgG ELISA was performed on 8-week sera to evaluate anti-HA antibody responses. Differences between monovalent and multivalent vaccines were evaluated by Mann-Whitney analysis; A strains $P=0.639, P=0.361$ for $\mathrm{B}$ strains, two tailed. b Intra-nasal administration of Ad-HA vaccines in BALB/c mice. For the blend of 1:1:1 and for all single-dosed vaccine groups animals were dosed at $2 \times 10^{6} \mathrm{IU}$ at 0 and 4 weeks. For the 1:10:1 blend Ad-H1 and $\mathrm{H} 3$ were dosed at $2 \times 10^{6}$ $\mathrm{IU}$, and $\mathrm{B}$ virus at $2 \times 10^{7} \mathrm{IU}$. Sera were harvested at 3 weeks and an anti-HA IgG ELISA was performed. $N=6 /$ group for all groups, except $N=5$ for $1: 10: 1$ blended group. Ad adenovirus, $C I$ confidence interval, ELISA enzyme-linked immunosorbent assay, GMT geometric mean titer, $H A$ hemagglutinin, $\operatorname{Ig} G$ immunoglobulin, i.n. intra-nasal, p.o. peroral

\section{Endoscopic Delivery of Bivalent B Vaccine Blend in Ferrets}

Monovalent and bivalent Ad-B/Wis and Ad-B/ Bris vaccine compositions were tested by the endoscopic route of delivery (p.o.) in ferrets as a model of oral tablet delivery in humans. As a 


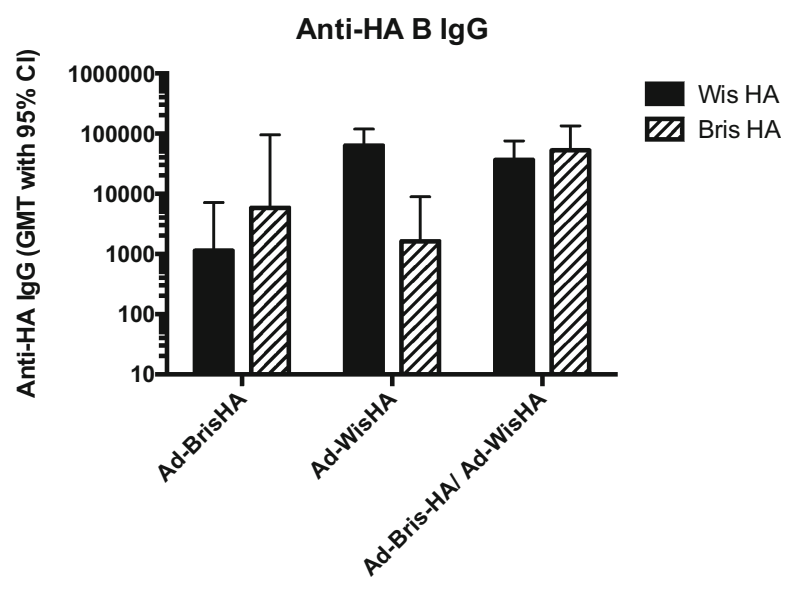

Fig. 3 Cross reactivity of B/Victoria (Bris) and $\mathrm{B} /$ Yamagata (Wis) HA antigens post-i.n. vaccination with $\mathrm{Ad}-\mathrm{B} / \mathrm{Bris}$, $\mathrm{Ad}-\mathrm{B} / \mathrm{W}$ is or a $1: 1$ blend of both vectors. $\mathrm{BALB} / \mathrm{c}$ mice were vaccinated with $2 \times 10^{6} \mathrm{IU}$ of Ad-Wisconsin/1/10 HA or Ad-Brisbane/60/08 HA or a blend of both at $2 \times 10^{6} \mathrm{IU}$ for each strain. Sera were harvested 3 weeks post-vaccination and anti-HA IgG ELISA was performed. $N=6 /$ group for all groups. $A d$ adenovirus, $C I$ confidence interval, ELISA enzyme-linked immunosorbent assay, GMT geometric mean titer, $H A$ hemagglutinin, $\operatorname{Ig} G$ immunoglobulin, i.n. intra-nasal

control for delivery, monovalent vectors were also given by i.m. injection using the same dose as p.o. As was seen in mice, both vectors elicited some cross reactivity to the homologous antigen and to the cross lineage antigen (Fig. 4). As an example, the Wisconsin HA-treated animals generated a similar antibody response to both $\mathrm{B} / \mathrm{Wisconsin} / 1 / 10$ and $\mathrm{B} / \mathrm{Brisbane} / 60 / 08 \mathrm{HA}$ proteins as measured by ELISA. The Ad-B/Bris immunized animals had a substantial response to $\mathrm{B} / \mathrm{Brisbane} / 60 / 08$ $\mathrm{HA}$ but the response to $\mathrm{B} / \mathrm{Wisconsin} / 1 / 10 \mathrm{HA}$ was quite varied with some animals having a much reduced response compared to the Ad-B/ Wis vaccine. The blended B-treated animals generated a similar antibody response to both HA types with GMTs of $1.22 \times 10^{4} \pm 3.43 \times 10^{4}$ and $1.22 \times 10^{4} \pm 3.5 \times 10^{4}$, respectively (Fig. 4).

In addition to total antibody titers, HAI and $\mathrm{MN}$ assays were performed to detect antibodies that could either prevent hemagglutination (Fig. 4b) or those that could neutralize influenza viruses (Fig. 4c). HAI assays were performed for all groups to both Brisbane and Wisconsin influenza viruses. Unlike total anti-HA antibodies, there was very little cross HAI antibody activity as is expected from the fact that human vaccines to either strain do not generate cross lineage protection. The response to $\mathrm{B} / \mathrm{Brisbane} / 60 / 08$ trended lower for Ad-B/ Bris, i.m. $160 \pm 170$ and p.o. $6 \pm 4(\mathrm{GMT} \pm \mathrm{SD})$ than the $\mathrm{B} / \mathrm{Wisconsin} / 1 / 10$ response for the Ad-B/Wis vaccines by the same routes of administration (806 \pm 370 i.m.; $62 \pm 65$ p.o.). There was a lot of variability in the Ad-B/Bris i.m.-treated animals to B/Brisbane/60/08 which accounts for the extremely large error bars when plotted GMT plus 95\% CI. Three of eight animals in the Ad-B/Bris p.o. group had titers $\geq 40$ (320, 40, and 160) compared to 6/8 with titers $\geq 40$ for the Ad-B/Wis p.o. group. Eight of eight animals in the Ad-B/Wis p.o. group had a $4 \times$ rise in titer (i.e., titers $\geq 20$ ) compared to $3 / 8$ for $B / B$ risbane $/ 60 / 08$. For the blended group, the GMT HAI for B/Wisconsin/1/10 was $21 \pm 53$ and for $\mathrm{B} / \mathrm{Brisbane} / 60 / 08$ was $15 \pm 10$. For $\mathrm{B} /$ Wisconsin/1/10, 3/8 animals had titers $\geq 40$ $(80,160$, and 40$)$ and 5 out of 8 had titers $(\geq 20)$. For B/Brisbane/60/08 only one animal had a titer $\geq 40$ but $4 / 8$ animals had a titer $\geq 20$, indicating a $4 \times$ rise in titer. There was no significant difference between the individual and blended p.o. groups for eliciting B/Wisconsin/1/10 HAI titers $(P=0.1$ by Mann-Whitney analysis) or the frequency at eliciting a fourfold $(4 \times)$ rise in HAI titers ( $P=0.3$ by Fischer's exact test).

$\mathrm{MN}$ data trended in a similar fashion to HAI's with Ad-B/Wis eliciting a stronger response compared to Ad-B/Bris for both i.m. and p.o., respectively (Fig. 4c). The anti-B/Wisconsin/1/ 10 GMT for Ad-B/Wis i.m. was $871 \pm 332$, while 
A
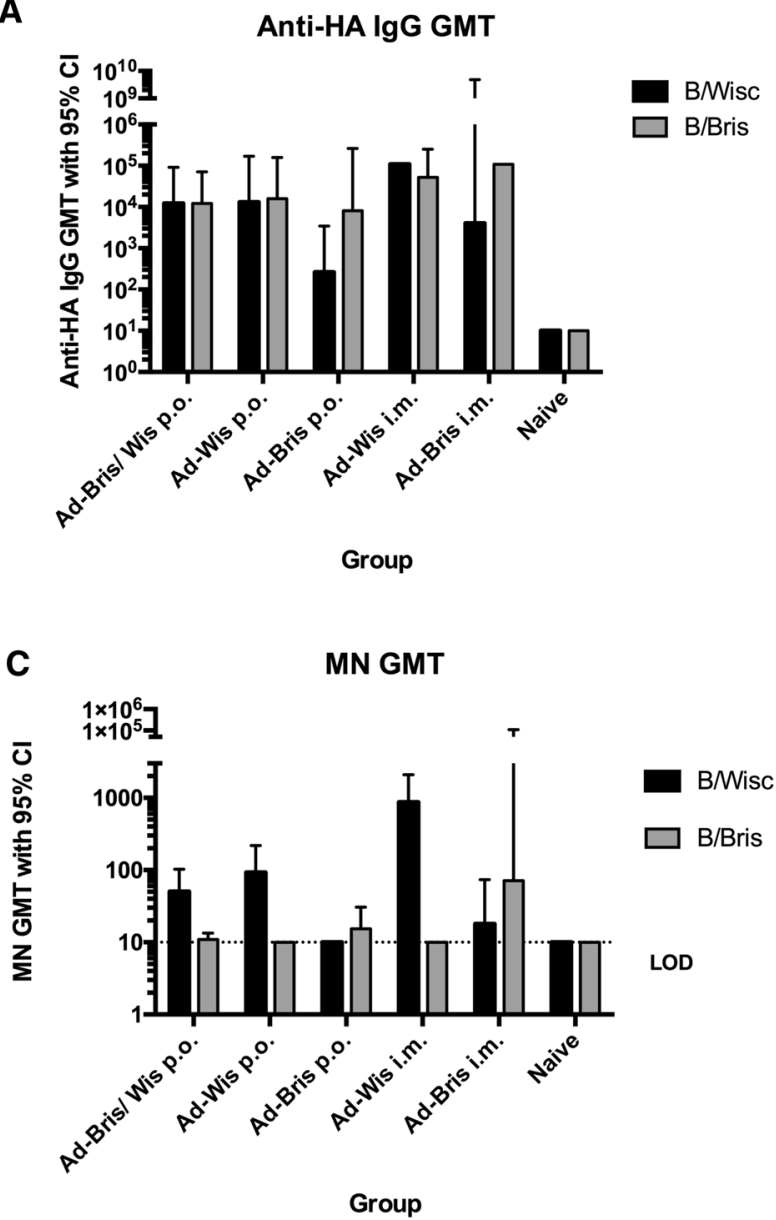

Fig. 4 Evaluation of immunogenicity to $B$ vaccine vectors in ferrets. $\mathrm{Ad}$ vectors expressing $\mathrm{B} / \mathrm{Brisbane} / 60 / 08$ (Ad-B/Wis) or $\mathrm{B} / \mathrm{W}$ isconsin/1/10 (Ad-B/Bris) or a $1: 1$ blend of each were evaluated in ferrets. For the monovalent vectors the vaccines were tested by p.o and i.m. routes of administration. All vectors were dosed at $1 \times 10^{9} \mathrm{IU} /$ vector/ferret at day 0 and day 28. Day 56 data for (a) total antibody by anti-HA IgG ELISA (b) HAI or (c) data MN are shown for both antigens. $N=8$ for p.o. and naïve

for Ad-B/Bris i.m. the anti-B/Brisbane/60/08 response was $80 \pm 85$ at 8 weeks post the first vaccination. For the p.o. Ad-B/Wis group the GMT B/Wisconsin/1/10 MN was $93 \pm 106$ while for the Ad-B/Bris p.o. group the B/Brisbane/60/ $08 \mathrm{MN}$ was $15 \pm 26.87 .5 \%$ of the p.o Ad-B/Wis group had a $\mathrm{B} / \mathrm{Wisconsin} / 1 / 10$ titer $\geq 40$ and the same percentage was $\geq 20$. For the blended

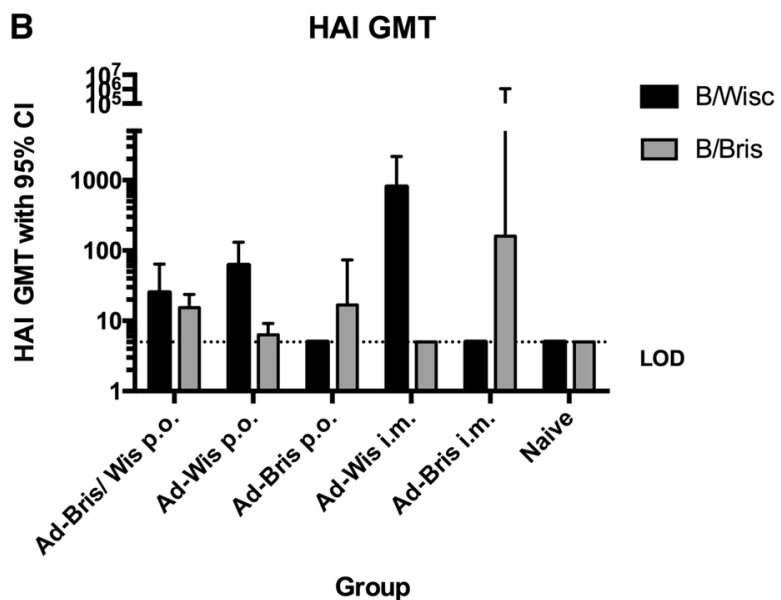

groups, $N=3$ for Ad-B/Wis, $N=2$ for Ad-B/Bris i.m. groups. Data are plotted for GMT $+95 \%$ CI which accounts for the large error bars for $N=2$ group. Ad adenovirus, $C I$ confidence interval, ELISA enzyme-linked immunosorbent assay, GMT geometric mean titer, $H A$ hemagglutinin, $H A I$ hemagglutinin inhibition, i.m. Intra-muscular, $I g G$ immunoglobulin, $L O D$ limit of detection, $M N$ microneutralization, p.o. peroral

group, $62.5 \%$ had anti-B/Wisconsin/1/10 MN titers $\geq 40$ (a $4 \times$ increase) and $87.5 \%$ had titers $\geq 20$. The Ad-B/Bris groups (monovalent and blended) induced low levels of B/Brisbane/60/ $08 \mathrm{MN}$ titers, with 25 and $12.5 \%$ of the mono and blended groups with titers $\geq 20$. MN titers for H3/Perth are not shown due to high background in the assay. 


\section{Endoscopic Delivery of Quadrivalent}

Vaccine Blend in Ferrets and Comparison

to i.m. Delivery of an Approved

Inactivated Influenza Vaccine

Monovalent A and B, bivalent A, and quadrivalent influenza vaccines were administered after endoscope delivery. Each animal was given $1 \times 10^{9} \mathrm{IU}$ of each vector. Total anti-HA antibody at 8 weeks is presented in Fig. 5a. The percent responders are shown in Table 1. Nearly all animals in all groups
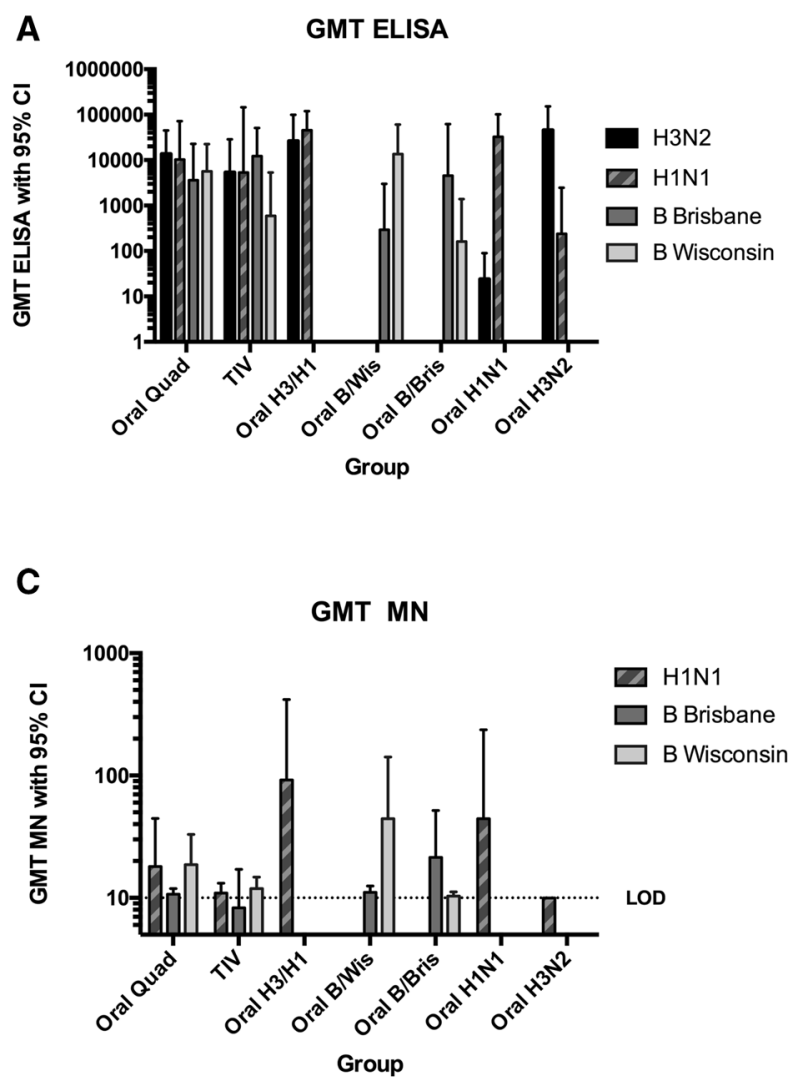

Fig. 5 Evaluation of immunogenicity to quadrivalent vectors in ferrets. Ad vectors expressing B/Brisbane/60/08, $\mathrm{B} / \mathrm{Wisconsin} / 1 / 10, \mathrm{~A} / \mathrm{H} 3 /$ Perth/16/09, and $\mathrm{A} / \mathrm{H} 1 /$ California/04/09 were evaluated in ferrets either as monovalent vaccines or as a divalent $\mathrm{A}$ blend $(\mathrm{A} / \mathrm{H} 3$ and $\mathrm{A} / \mathrm{H} 1)$ or as a quadrivalent blend $(\mathrm{B} / \mathrm{Bris}$ and $\mathrm{B} / \mathrm{W}$ is, $\mathrm{A} / \mathrm{H} 3$ and $\mathrm{A} / \mathrm{H} 1)$. All vectors were dosed at $1 \times 10^{9} \mathrm{IU} /$ vector/ferret. Sera were analyzed at day 56. (a) Total antibody by anti-HA IgG ELISA (b) HAI or (c) MS data are shown for responded to the corresponding $\mathrm{HA}$ of the administered vaccine with the exception of two animals (20\%) in the Ad-B/Bris group (Group 2) and one animal (10\%) in the quadrivalent blended group (Group 6); these animals failed to generate anti-H1 HA IgG responses. There was substantial cross reactivity within the influenza $A$ and $B$ vaccine groups with $60 \%$ of Ad-B/Wis-treated animals reacting against $\mathrm{B} /$ Brisbane/60/08 $\mathrm{HA}$ and 50\% of Ad-B/Bris-treated animals reacting with B/Wisconsin/1/10 HA. Similarly, $50 \%$ of
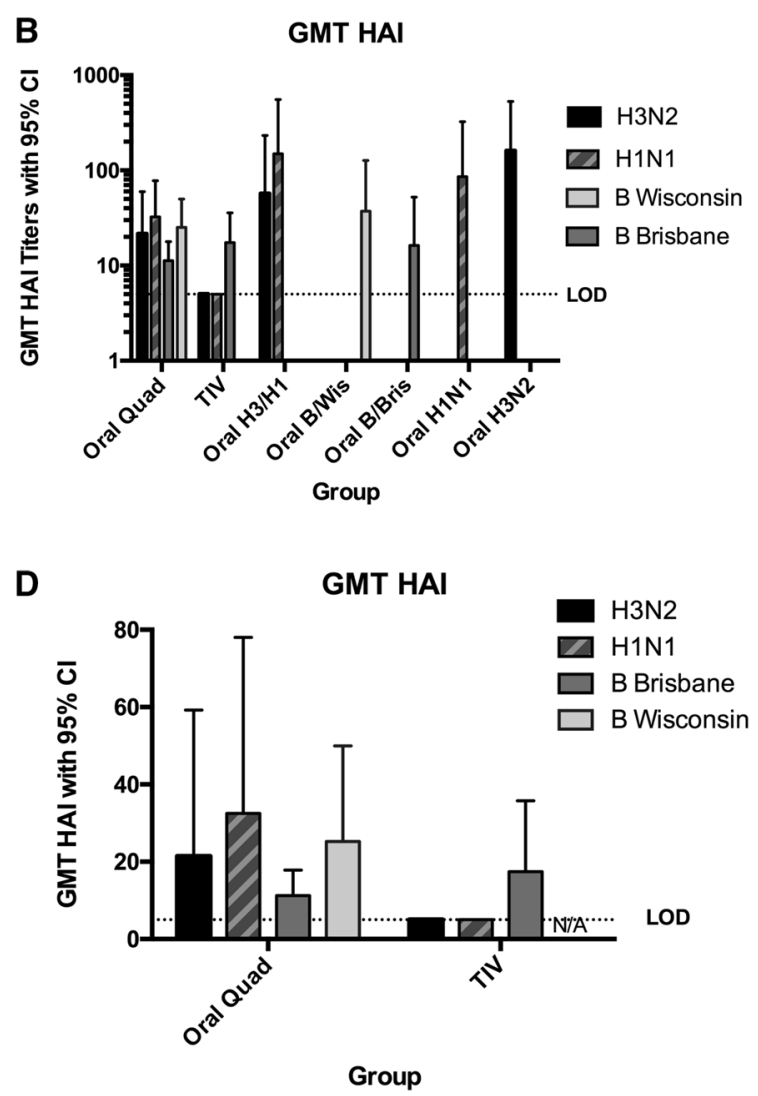

the different antigen strains. Note no H3 MN data are available due to high background in the assay. d Direct comparison between quadrivalent oral vaccine versus TIV. $N=10$ for Ad vaccine groups. $N=4$ for TIV vaccine. $A d$ adenovirus, $C I$ confidence interval, ELISA enzyme-linked immunosorbent assay, GMT geometric mean titer, $H A$ hemagglutinin, $H A I$ hemagglutinin inhibition, $I g G$ Immunoglobulin, $L O D$ limit of detection, $M N$ microneutralization, $T I V$ trivalent inactivated influenza vaccine 
Table 1 Total antibody responders in monovalent and multivalent groups in ferrets

\begin{tabular}{lllll}
\hline Group & \multicolumn{2}{l}{ \% anti-HA IgG responders } & \\
\cline { 2 - 5 } & B/Wisconsin/1/10 & B/Brisbane/60/08 & H3/Perth/16/09 & H1/California/07/09 \\
\hline Ad-Wis & 100 & 60 & ND & ND \\
Ad-Bris & 50 & 70 & ND & ND \\
Ad-Perth & ND & ND & 100 & 60 \\
Ad-CA409 & ND & ND & 20 & 100 \\
Ad-Perth/Ad-CA409 & ND & ND & 100 & 100 \\
Ad-Wis/Bris/Perth/CA409 & 100 & 100 & 100 & 90 \\
TIV & N/A & 100 & 100 & 100 \\
\hline
\end{tabular}

$N / A$ not applicable, $N / D$ not done, $T I V$ trivalent inactivated influenza vaccine

Ad-H3-vaccinated animals reacted against H1 HA though only $20 \%$ of Ad-H1 reacted against H3. There was no significant difference in total anti-HA IgG titers between the monovalent versus quadrivalent, and the quadrivalent versus TIV groups as tested by Mann-Whitney analysis $(P=0.08-0.99)$, with the exception that the quadrivalent was significantly better for H3 than TIV $(P=0.02)$.

HAI analysis was performed for all groups. The HAI titers were not significantly different between quadrivalent and monovalent vaccine groups $(P=0.22-0.96)$ with the exception of the H3 monovalent versus the quadrivalent which indicated that the monovalent induced a higher $\mathrm{H} 3 \mathrm{HAI}$ response (Fig. $5 \mathrm{~b} ; P=0.02$ by Mann-Whitney analysis).

$\mathrm{MN}$ data trended similar to HAI titers though the percent responders and the absolute geometric titers were in general lower (Fig. 5c) for all groups including the TIV group which elicited no MN titers greater than $2 \times$ the assay background of 10 . Only $20 \%$ of the quadrivalent group responded ( $4 \times$ above background) to H1N1 and B/Wisconsin/ $1 / 10$ (Table 2). This increased to $40 \%$ responders for $\mathrm{B} /$ Wisconsin/1/10 if a $2 \times$ above background criteria was used. There were no $\mathrm{MN}$ titers detected to $\mathrm{B} /$ Brisbane/60/08, which is consistent with our experience in that this HA appears to be less immunogenic than other HAs tested.

A direct head to head comparison between TIV and the oral quadrivalent (Oral Quad) vaccine for HAI is shown in Fig. 5d. TIV was given as a full human dose $(45 \mu \mathrm{g})$ protein. GMT HAI at 8 weeks was higher for the $\mathrm{H} 3$ and $\mathrm{H} 1$ components compared to TIV. The TIV failed to elicit anti-H1 or H3 HAI responses despite the fact that these animals had generated a relatively strong total HA antibody response as measured by ELISA. The proportion of $2 \times$ HAI responders was significantly better with the oral Quad than TIV for H1 HAI and trended higher for H3 HAI $(P=0.001, P=0.07$ by Fisher's exact test), and trended higher for $4 \times$ HAI responses $(P=0.07, P=0.23$ by Fisher's exact test). Table 3 has detailed these comparisons. The Ad-B/ Bris monovalent (GMT $16.25 \pm 73.98$ ) and the quadrivalent vaccine $(11.23 \pm 10.59)$ had about the same anti-B HAI response as TIV $(16.82 \pm 14.14 ;$ Fig. $5 b$, d). TIV had a similar number of $\mathrm{B} /$ Brisbane/60/08 responders compared to the quad vector, 50 versus $40 \%$ for $4 \times$ above background (background is 5), 100 versus $40 \%$ for $2 \times$ above background (Table 2 ). As there is no $\mathrm{B} /$ Yamagata component in TIV, the anti-Wisconsin/1/10 HAI response cannot be compared. 
Table 2 Total MN responders in monovalent and multivalent groups in ferrets

\begin{tabular}{lllll}
\hline Group & \multicolumn{2}{l}{ \% MN responders } & & \\
\cline { 2 - 5 } & B/Wisconsin/1/10 & B/Brisbane/60/08 & H3/Perth/16/09 & H1/California/07/09 \\
\hline Ad-Wis & $40(50)$ & 0 & 0 & 0 \\
Ad-Bris & 0 & $30(30)$ & 0 & 0 \\
Ad-Perth & 0 & 0 & N/A & 0 \\
Ad-CA409 & 0 & 0 & N/A & $30(30)$ \\
Ad-Perth/Ad-CA409 & ND & ND & N/A & $60(60)$ \\
Ad-Wis/Bris/Perth/CA409 & $20(40)$ & 0 & N/A & $20(20)$ \\
TIV & N/A & 0 & N/A & 0 \\
\hline
\end{tabular}

Percentage responders with fourfold increase in titer are shown. The percentage in () represents responders with a twofold increase in titer

$N / A$ not applicable, $N / D$ not done, $T I V$ trivalent inactivated influenza vaccine

Table 3 Total HAI responders in monovalent and multivalent groups in ferrets

\begin{tabular}{lllll}
\hline Group & \multicolumn{2}{l}{ \% HAI responders } & & \\
\cline { 2 - 5 } & B/Wisconsin/1/10 & B/Brisbane/60/08 & H3/Perth/16/09 & H1/California/07/09 \\
\hline Ad-Wis & $60(90)$ & ND & ND & ND \\
Ad-Bris & ND & $40(40)$ & ND & ND \\
Ad-Perth & ND & ND & $90(90)$ & ND \\
Ad-CA409 & ND & ND & ND & $90(100)$ \\
Ad-Perth/Ad-CA409 & ND & ND & $60(90)$ & 100 \\
Ad-Wis/Bris/Perth/CA409 & $50(100)$ & $30(70)$ & $50(70)$ & $70(100)$ \\
TIV & N/A & $50(100)$ & 0 & 0 \\
\hline
\end{tabular}

Percentage responders with fourfold increase in titer are shown. The percentage in () represents responders with a twofold increase in titer

$N / A$ not applicable, $N / D$ not done, $T I V$ trivalent inactivated influenza vaccine

${ }^{a} \mathrm{~N} / \mathrm{A}$ as Fluzone does not have a vaccine component against $\mathrm{B} / \mathrm{Wis} / 1 / 10$ or any other Yamagata strain

\section{DISCUSSION}

Seasonal influenza vaccines have advanced to provide greater protection against multiple flu strains. The original influenza vaccines were monovalent vaccines against single influenza subtypes [16]; however, due to co-circulation of multiple strains and the unpredictable nature of which strain might become the dominant strain in an influenza season, influenza vaccines have become more complex to account for these risks. This evolution led to the development and approval of quadrivalent influenza vaccines in the last four years (Fluarix ${ }^{\circledR}$ Quadrivalent, GlaxoSmithKline Biologicals; FluLaval $^{\left({ }^{\circledR}\right.}$ Quadrivalent, ID Biomedical Corp; Fluzone $^{\circledR}$ Quadrivalent, Sanofi Pasteur Inc.); and FluMist ${ }^{\circledR}$ Quadrivalent, Medimmune, LLC) [36]. This 
development has also been fueled by improvements in the capacity of production, which can accommodate these additional components. Additional improvements in technology have led to further advances in influenza vaccines. In 2012, the first cell culture-derived influenza vaccine, Flucelvax ${ }^{\circledR}$ (Novartis Vaccines \& Diagnostics, Inc.), rather than egg-derived vaccine, was approved, which can bypass potential limitations on egg availability. A number of novel subunit approaches based on injectable HAs have or are in the process of being commercialized and include the use of baculovirus-derived HA, Flublock $^{\circledR}$ (Protein Sciences Corporation), baculovirus-derived influenza vaccine-like particles [17], or the use of bacterial expressed flagellin-HA chimeras as injectable vaccines [18]. Similarly, our approach is a departure from traditional egg-derived influenza vaccines, using cell culture-derived adenovirus as a vector. Instead of injecting a subunit of HA, our approach delivers a vector that co-expresses influenza HA and a dsRNA adjuvant to improve immune recognition of the HA [6]. We have delivered vaccine to humans in tablet or in liquid form, with the tablet showing slightly better HAI responses (Liebowitz et al. [8] and Kim et al., unpublished data). The current study demonstrates that this platform can be used to generate multivalent adenovirus vaccines, and is achieved by blending multiple monovalent vaccine vectors. This study is unique as it is the first published study using adenoviruses as vaccine vectors for a quadrivalent influenza vaccine and using an oral model of delivery.

This study primarily demonstrates that a blended quadrivalent vaccine is effective at inducing antibody responses with minimal interference to four influenza $\mathrm{HA}$ antigens (H1, H3, B/Victoria, and B/Wisconsin) after endoscopic delivery in ferrets. To model a quadrivalent tablet vaccine, all four monovalent vaccines were mixed and delivered to the same location in the ferret intestine by endoscope. In this study, the quadrivalent vaccine induced $90-100 \%$ responders by ELISA, which was comparable to the percent responders induced for the monovalent vectors (70-100\%) and 30-70\% responders with a $4 \times$ rise in HAI for the quad vaccines (70-100\%) responders with a $2 \times$ rise. This immunogenicity compares well to the immunogenicity of commercial vaccines in humans [37]. We expect the vaccine would provide protection even in the absence of HAI responses as we previously demonstrated that protection against $\mathrm{H} 5 \mathrm{~N} 1$ using this platform correlated with total antibody and not HAI [6]. The rAd doses tested in ferrets in this study were relatively low (1-10\%) compared to human doses where we have seen substantial immune responses in clinical trials $[8,9]$ and compare well with the immune response generated to a full human dose of TIV that we evaluated concurrently. The HA protein made from a gene-based vaccine, such as ours, cannot be measured because it is made inside each subject or animal, so the best way to compare is in equivalent human doses.

Of interest was the fact that the TIV vaccine at a full human dose, by the recommended route of delivery, only induced HAI responses to $\mathrm{B} /$ Brisbane, and no HAI responses to $\mathrm{A} / \mathrm{H} 1 \mathrm{~N} 1$ or A/H3N2. A published study by Skowronski et al. [19] has also noted that TIV vaccines are poor HAI inducers in influenza-naive ferrets. Pearce et al. [20] found in ferrets that the best HAI response in the TIV was to B/Brisbane/60/08, (Titers 20-80) after a single immunization and generated a low HAI response to the other seasonal components after two immunizations. In contrast, our vaccine induced HAI responses to all antigens and a similar response to the 
B/Brisbane/60/08 antigen as TIV. A major question is what accounts for the difference in HAI observed in ferrets between our vaccine and the tested TIV. As the ferrets used in these studies are screened and selected for animals that have negative HAI titers to major influenza strains, influenza vaccine studies in ferrets are essentially priming studies. Given that the results obtained with $1: 100\left(1 \times 10^{9} \mathrm{IU}\right)$ of a rAd human dose $\left(1 \times 10^{11} \mathrm{IU}\right)$ were equal or better than a full TIV dose in inducing HAI responses, it is possible that rAd makes for a superior priming vaccine in naïve individuals. One possible explanation for the priming difference is that a vector-based approach elicits a superior $\mathrm{T}$ cell response (reviewed in $[21,22])$, and that this can improve HAI titers in the absence of pre-exposure to the matched influenza strain. Several investigators have demonstrated the ability of adenovirus to induce strong $\mathrm{T}$ cell responses in animals and humans $[9,23]$. Observations for the role of $\mathrm{T}$ cell help in improved vaccine responses have been made for $\mathrm{H} 5$ and $\mathrm{H} 1$ pandemic strains in humans, where enhanced CD4 $\mathrm{T}$ cell responses to HA promoted higher HAI titers [24, 25]. One possibility is that the effector $\mathrm{T}$ cell help induced at each immunization provides for higher antibody responses at each time point so the boost is much more effective. The other possibility is that memory $\mathrm{CD} 4 \mathrm{~T}$ cell responses induced after the first immunization allows for better HAI responses upon the second immunization. $\mathrm{T}$ cell responses in ferrets are not easy to measure, so careful measurements of $\mathrm{T}$ cell responses and HAI post-immunization will need to be performed in HAI-naïve humans to demonstrate priming potential.

The approach we have taken of co-delivering a blend of four vaccine vectors to a specific location in the ferret intestine proved effective at eliciting immune responses, but the use of
rAd in a tablet format allows for alternative approaches. For delivery to humans we could take a similar approach to the ferret study by blending all four strains together in a single tablet. Alternatively, a quadrivalent vaccine could be administered as four tablets with each tablet delivering a vaccine for one of the targeted quadrivalent strains. The advantage of this single strain per tablet approach is that if late season changes were needed on one strain, the other three strain tablets would not be impacted. Each strain would be individually released and kept, and all four vaccines could be handed out at once or given as they were made. It would be difficult to take the same approach with an injected vaccine because taking four separate shots for influenza would be difficult to sell, and if all strains were blended together, all components would need to be discarded after a single strain change. An additional advantage of the rAd-based approach is that multiple antigens could fit on one vector. This was demonstrated for a tetravalent adenovirus dengue vaccine generated by a combination of blending and expressing multiple antigens on the same adenovirus backbone, and provided significant protection to four dengue serotypes in rhesus monkeys [26]. We have explored a similar approach and have expressed multiple genes including two HA genes on single $\mathrm{Ad}$ vectors using the foot and mouth $2 \mathrm{~A}$ linker sequence, which permits the expression of multiple transcripts from a single ORF ([27] and data not shown). This approach has the advantage of reducing the production requirements of the individual vector components.

Pre-existing immunity to adenovirus, or to any vector, can be problematic when using that vector to deliver and express a vaccine antigen. In studies by Ledgerwood et al. [28], an adenovirus used to express an Ebola virus 
antigen was not as potent in humans with pre-existing neutralizing antibody responses to the adenovirus as in humans without neutralizing antibody responses. One method to potentially circumvent this problem is to choose a vector with low seroprevalence in humans such as simian adenoviruses or engineered chimeric adenoviruses [29, 30]. There are problems with this novel vector approach in that not all vectors are equally immunogenic as adenovirus type 5, and once the vaccine vector is used in a human, that vector will not be useful for a new indication or a boost. Injected adenoviruses elicit a very strong anti-vector response that can block subsequent uses or administrations. The situation would get extremely complicated if adenoviral vectors were in wide-scale human use, and vaccine developers were forced to screen people for specific antibody titers, before deciding whether the vaccine could be given. A different alternative is to deliver adenoviruses orally, which appears to circumvent neutralizing antibody responses and selectively elicit anti-transgene immune responses (not anti-vector antibody responses) $[6,31]$. In our human tablet study, pre-existing immunity to adenovirus 5 had no effect on the ability to elicit HAI responses; the subject with the highest HAI response to the vaccine also had the highest pre-existing anti-adenovirus neutralizing antibody response [8].

Future human trials will likely follow a similar path as our ferret studies, first testing A and $\mathrm{B}$ monovalent vaccines and then testing combinations to evaluate efficacy and possible interference effects. Multivalent approaches using either DNA or viral vectors such as Ad are not new. A DNA vaccination approach in mice using blends of HA expressing plasmids against multiple $\mathrm{H} 3$ strains increased the breath of protection to multiple strains compared to monovalent vaccines alone [32]. Blends of adenovirus vaccine vectors expressing HIV antigens have previously been tested in animals without signs of immunological interference between vaccine antigens [33]. Given our success of this oral platform in humans, plus the encouraging response described here in ferrets, it is our intention to evaluate such blends in future human trials. Only through clinical trials can it be determined whether such multivalent vaccines will prove efficacious as vaccines.

\section{ACKNOWLEDGMENTS}

Sponsorship of this study and article processing charges were funded by Vaxart, Inc. All named authors meet the International Committee of Medical Journal Editors (ICMJE) criteria for authorship of this manuscript, take responsibility for the integrity of the work as a whole, and have given final approval to the version to be published. Thanks to James Toomey, Logan Haller, and Diana Noah at Southern Research Institute for help with the ferret vaccine studies.

Disclosures. Ciaran D. Scallan, Jonathan D. Lindbloom, and Sean N. Tucker are either employees of Vaxart, inc. and/or hold stock options in Vaxart. No outside writers provided assistance in writing the manuscript.

Compliance with Ethics Guidelines. All institutional and national guidelines for the care and use of laboratory animals were followed. 
Open Access. This article is distributed under the terms of the Creative Commons Attribution-NonCommercial 4.0 International License (http://creativecommons.org/licenses/ by-nc/4.0/), which permits any noncommercial use, distribution, and reproduction in any medium, provided you give appropriate credit to the original author(s) and the source, provide a link to the Creative Commons license, and indicate if changes were made.

\section{REFERENCES}

1. Ambrose CS, Levin MJ. The rationale for quadrivalent influenza vaccines. Hum Vaccin Immunother. 2012;8:81-8.

2. Belshe RB. The need for quadrivalent vaccine against seasonal influenza. Vaccine. 2010;28(Suppl 4):D45-53.

3. Van Bellinghen LA, Meier G, Van Vlaenderen I. The potential cost-effectiveness of quadrivalent versus trivalent influenza vaccine in elderly people and clinical risk groups in the UK: a lifetime multi-cohort model. PLoS One. 2014;9:e98437.

4. Potter CW, Jennings R, Clark A, Ali M. Interference following dual inoculation with influenza A (H3N2) and (H1N1) viruses in ferrets and volunteers. J Med Virol. 1983;11:77-86.

5. Romanova JR, Ermachenko TA, Alexandrova GI, Tannock GA. Interference between cold-adapted (ca) influenza $\mathrm{A}$ and $\mathrm{B}$ vaccine reassortants or between ca reassortants and wild-type strains in eggs and mice. Vaccine. 1994;12:23-7.

6. Scallan CD, Tingley DW, Lindbloom JD, Toomey JS, Tucker SN. An adenovirus-based vaccine with a double-stranded RNA adjuvant protects mice and ferrets against H5N1 avian influenza in oral delivery models. Clin Vaccine Immunol. 2013;20:85-94.

7. Hobson D, Curry RL, Beare AS, Ward-Gardner A. The role of serum haemagglutination-inhibiting antibody in protection against challenge infection with influenza A2 and B viruses. J Hyg (Lond). 1972;70:767-77.

8. Liebowitz D, Lindbloom JD, Brandl JR, Garg SJ, Tucker SN. High titer neutralizing antibodies to influenza following oral tablet immunization: a randomized. Placebo-controll Trial Lancet Infect Dis. $2015 ; 15: 1041-8$.

9. Peters W, Brandl JR, Lindbloom JD, Martinez CJ, Scallan CD, Trager GR, Tingley DW, Kabongo ML, Tucker SN. Oral administration of an adenovirus vector encoding both an avian influenza A hemagglutinin and a TLR3 ligand induces antigen specific granzyme $B$ and IFN-gamma $T$ cell responses in humans. Vaccine. 2013;31:1752-8.

10. Kong Q, Richter L, Yang YF, Arntzen CJ, Mason HS, Thanavala Y. Oral immunization with hepatitis B surface antigen expressed in transgenic plants. Proc Natl Acad Sci USA. 2001;98:11539-44.

11. Sharpe S, Fooks A, Lee J, Hayes K, Clegg C, Cranage M. Single oral immunization with replication deficient recombinant adenovirus elicits long-lived transgene-specific cellular and humoral immune responses. Virology. 2002;293:210-6.

12. Moore AC, Kong WP, Chakrabarti BK, Nabel GJ. Effects of antigen and genetic adjuvants on immune responses to human immunodeficiency virus DNA vaccines in mice. $J$ Virol. 2002;76:243-50.

13. Holman DH, Wang D, Raja NU, Luo M, Moore KM, Woraratanadharm J, Mytle N, Dong JY. Multi-antigen vaccines based on complex adenovirus vectors induce protective immune responses against $\mathrm{H} 5 \mathrm{~N} 1$ avian influenza viruses. Vaccine. 2008;26:2627-39.

14. Monto AS, Maassab HF. Ether treatment of type B influenza virus antigen for the hemagglutination inhibition test. J Clin Microbiol. 1981;13:54-7.

15. Rowe T, Abernathy RA, Hu-Primmer J, Thompson WW, Lu X, Lim W, Fukuda K, Cox NJ, Katz JM. Detection of antibody to avian influenza A (H5N1) virus in human serum by using a combination of serologic assays. J Clin Microbiol. 1999;37:937-43.

16. Plotkin SL, Plotkin SA. A short history of vaccination. In: Plotkin SA, editor. Vaccines. P.A., Orenstein: W.A. \& Offit; 2013. p. 1-13.

17. Fries LF, Smith GE, Glenn GM. A recombinant viruslike particle influenza A (H7N9) vaccine. N Engl J Med. 2013;369:2564-6.

18. Song L, Liu G, Umlauf S, Liu X, Li H, Tian H, Reiserova L, Hou F, Bell R, Tussey L. A rationally designed form of the TLR5 agonist, flagellin, supports superior immunogenicity of Influenza B globular head vaccines. Vaccine. 2014;32: 4317-23.

19. Skowronski DM, Hamelin ME, De Serres G, Janjua NZ, Li G, Sabaiduc S, Bouhy X, Couture C, Leung A, 
Kobasa D, Embury-Hyatt C, de Bruin E, Balshaw R, Lavigne S, Petric M, Koopmans M, Boivin G. Randomized controlled ferret study to assess the direct impact of 2008-09 trivalent inactivated influenza vaccine on $\mathrm{A}(\mathrm{H} 1 \mathrm{~N} 1) \mathrm{pdm} 09$ disease risk. PLoS ONE. 2014;9:e86555.

20. Pearce MB, Belser JA, Gustin KM, Pappas C, Houser KV, Sun X, Maines TR, Pantin-Jackwood MJ, Katz JM, Tumpey TM. Seasonal trivalent inactivated influenza vaccine protects against 1918 Spanish influenza virus infection in ferrets. J Virol. 2012;86:7118-25.

21. Draper SJ, Heeney JL. Viruses as vaccine vectors for infectious diseases and cancer. Nat Rev Microbiol. 2010;8:62-73.

22. Rocha CD, Caetano BC, Machado AV, Bruna-Romero O. Recombinant viruses as tools to induce protective cellular immunity against infectious diseases. Int Microbiol. 2004;7:83-94.

23. Draper SJ, Biswas S, Spencer AJ, Remarque EJ, Capone S, Naddeo M, Dicks MD, Faber BW, de Cassan SC, Folgori A, Nicosia A, Gilbert SC, Hill AV. Enhancing blood-stage malaria subunit vaccine immunogenicity in rhesus macaques by combining adenovirus, poxvirus, and protein-in-adjuvant vaccines. J Immunol. 2010;185:7583-95.

24. Galli G, Medini D, Borgogni E, Zedda L, Bardelli M, Malzone C, Nuti S, Tavarini S, Sammicheli C, Hilbert AK, Brauer V, Banzhoff A, Rappuoli R, Del Giudice G, Castellino F. Adjuvanted H5N1 vaccine induces early CD4 $+\mathrm{T}$ cell response that predicts long-term persistence of protective antibody levels. Proc Natl Acad Sci USA. 2009;106:3877-82.

25. Nayak JL, Fitzgerald TF, Richards KA, Yang $H$, Treanor JJ, Sant AJ. CD4 + T-cell expansion predicts neutralizing antibody responses to monovalent, inactivated 2009 pandemic influenza $\mathrm{A}(\mathrm{H} 1 \mathrm{~N} 1)$ virus subtype H1N1 vaccine. J Infect Dis. 2013;207:297-305.

26. Raviprakash K, Wang D, Ewing D, Holman DH, Block K, Woraratanadharm J, Chen L, Hayes C, Dong JY, Porter K. A tetravalent dengue vaccine based on a complex adenovirus vector provides significant protection in rhesus monkeys against all four serotypes of dengue virus. J Virol. 2008;82:6927-34.

27. Donnelly ML, Hughes LE, Luke G, Mendoza H, ten Dam E, Gani D, Ryan MD. The 'cleavage' activities of foot-and-mouth disease virus $2 \mathrm{~A}$ site-directed mutants and naturally occurring '2A-like' sequences. J Gen Virol. 2001;82:1027-41.
28. Ledgerwood JE, Costner P, Desai N, Holman L, Enama ME, Yamshchikov G, Mulangu S, Hu Z, Andrews CA, Sheets RA, Koup RA, Roederer M, Bailer R, Mascola JR, Pau MG, Sullivan NJ, Goudsmit J, Nabel GJ, Graham BS. A replication defective recombinant Ad5 vaccine expressing Ebola virus GP is safe and immunogenic in healthy adults. Vaccine. 2010;29:304-13.

29. Barouch DH, Liu J, Lynch DM, O'Brien KL, La Porte A, Simmons NL, Riggs AM, Clark S, Abbink P, Montefiori DC, Landucci G, Forthal DN, Self SG, Carville A, Mansfield K, Goudsmit J. Protective efficacy of a single immunization of a chimeric adenovirus vector-based vaccine against simian immunodeficiency virus challenge in rhesus monkeys. J Virol. 2009;83:9584-90.

30. Colloca S, Barnes E, Folgori A, Ammendola V, Capone S, Cirillo A, Siani L, Naddeo M, Grazioli F, Esposito ML, Ambrosio M, Sparacino A, Bartiromo M, Meola A, Smith K, Kurioka A, O'Hara GA, Ewer $\mathrm{KJ}$, Anagnostou N, Bliss C, Hill AV, Traboni C, Klenerman P, Cortese R, Nicosia A. Vaccine vectors derived from a large collection of simian adenoviruses induce potent cellular immunity across multiple species. Sci Transl Med. 2012;4:115ra112.

31. Xiang ZQ, Gao GP, Reyes-Sandoval A, Li Y, Wilson $\mathrm{JM}$, Ertl HC. Oral vaccination of mice with adenoviral vectors is not impaired by preexisting immunity to the vaccine carrier. J Virol. 2003;77:10780-9.

32. Huber VC, Thomas PG, McCullers JA. A multi-valent vaccine approach that elicits broad immunity within an influenza subtype. Vaccine. 2009;27:1192-200.

33. Casimiro DR, Cox K, Tang A, Sykes KJ, Feng M, Wang F, Bett A, Schleif WA, Liang X, Flynn J, Tobery TW, Wilson K, Finnefrock A, Huang L, Vitelli S, Lin J, Patel D, Davies ME, Heidecker GJ, Freed DC, Dubey S, O'Connor DH, Watkins DI, Zhang ZQ, Shiver JW. Efficacy of multivalent adenovirus-based vaccine against simian immunodeficiency virus challenge. J Virol. 2010;84:2996-3003.

34. Centers for Disease Control and Prevention. CDC Health Advisory Regarding the Potential for Circulation of Drifted Influenza A (H3N2) Viruses. http://emergency.cdc.gov/han/han00374. asp. Accessed 25 Mar 2016.

35. Centers for Disease Control and Prevention. Seasonal Influenza Vaccine Effectiveness, 2005-2015. http://www.cdc.gov/flu/professionals/ vaccination/effectiveness-studies.htm. Accessed 25 Mar 2016. 
36. Centers for Disease Control and Prevention. TABLE. Influenza vaccines-United States, 2015-16 influenza season. http://www.cdc.gov/flu/protect/ vaccine/vaccines.htm. Accessed 25 Mar 2016.

37. US Food and Drug Administration. FLUARIX QUADRIVALENT Prescribing Information.
http://www.fda.gov/downloads/BiologicsBloodVac cines/Vaccines/ApprovedProducts/UCM220624.pdf. Accessed 25 Mar 2016. 